Revista Estudiantil HUELLAS. Año 5 No. 10. (Febrero, 1990). Lenguas Modernas, Universidad Mariana. San Juan de Pasto.

APA citation style: Benavides B., Jorge E. (1990). Escucha y Habla vs. Texto en Inglés. Revista Huellas, Universidad Mariana, 5(10), 15-25

\title{
Escucha y Habla vs. Texto en Inglés
}

\author{
Jorge E. Benavides B. (joelbebu@gmail.com) \\ Departamento de Lingüística e Idiomas \\ Universidad de Nariño, San Juan de Pasto, Colombia
}

El presente artículo propone una reflexión dirigida tanto a estudiantes como a profesores del inglés quienes tienen a su cargo la tarea de aprender y guiar respectivamente en el dominio del idioma inglés. Mi propósito es el de dar a conocer un aspecto de interferencia de funciones en las estrategias de enseñanza-aprendizaje causada por la excesiva dependencia del texto en el área del desarrollo de habilidades en el idioma extranjero. En este caso está en tela de juicio la forma de aplicación de determinados ejercicios y prácticas en el desarrollo de la habilidad de escucha y producción oral comunicativa.

\section{Introducción}

No es desconocido en ninguna forma el concepto que existe y ha existido acerca de la baja producción oral comunicativa en el aprendizaje del inglés, inclusive hasta el punto de que este aspecto se tiene como algo inalcanzable en los niveles de educación secundaria, y por qué no decir, hasta los superiores. El hecho de desarrollar la habilidad de entender el inglés a nivel de escucha y hablarlo en forma fluida y satisfactoria no parece muy factible ya que la mayoría de profesores y estudiantes del inglés estamos empeñados en enseñar y aprender acerca del idioma y no el idioma como tal: A entenderlo y hablarlo funcionalmente. Es decir, nos hemos concentrado tal vez en el desarrollo de aspectos mal llamados pasivos de la lengua: la lectura, la comprensión de lectura, el vocabulario, la gramática, etc. Además, he podido constatar particularmente que en textos cuyo objetivo primordial está centrado en la función del idioma con propósito comunicativo (ejemplo la serie "In Touch", de moda en el momento) no están siendo utilizados adecuadamente precisamente en la parte activa del mismo o sea en las prácticas orales de escucha y de habla. Nuestra tendencia a enseñar acerca del idioma y no el idioma en sí se deja ver en el hecho de volver actividades de producción activas como la escucha y el habla en pasivas como en la escritura y la lectura de ejercicios que se supone 
Revista Estudiantil HUELLAS. Año 5 No. 10. (Febrero, 1990). Lenguas Modernas, Universidad Mariana. San Juan de Pasto.

están diseñados para desarrollar otro tipo de habilidades diferentes a las destrezas de acción comunicativa.

\section{El texto guía}

Es indudable que el texto de inglés es de gran utilidad en el campo del aprendizaje de un idioma extranjero en cualquiera de sus niveles. Sin embargo, esto no siempre es verdad si la función del texto y su uso no son los apropiados. Estoy refiriéndome concretamente al uso inadecuado y excesivo del texto de inglés en los primeros niveles de introducción en el campo de la habilidad comunicativa tanto de habla como de escucha. Estas son las actividades de introducción a la escucha, comprensión y producción oral en base al dialogo o historia foco de la lección y las actividades de escucha y habla que generalmente son las cinco o seis primeras actividades en cada una de las lecciones se la serie "In Touch".

Ante todo, para la parte oral, textos como los de "In Touch", o de ese estilo, en el que funciones comunicativas son el objetivo principal, las historias o diálogos presentados pueden hacer pensar tanto a los estudiantes como al profesor desprevenido que todo dialogo o ejercicio de escucha tiene que basarse en la lectura o en el peor de los casos en el de la memorización mediante la lectura.

Por el contrario, el propósito de la inclusión del dialogo o historia es el de presentar contenido contextual y comunicativo de las estructuras en cuestión. Son una guía para que el estudiante se ubique en lo posible mediante los personajes y el contexto en una situación pseudoreal y esto de pie a que las estructuras presentadas en los diálogos se realicen introduciendo el contexto real de experiencia de los estudiantes. Todo esto obviamente con el propósito de internalizar unos patrones a nivel de escucha y habla tendientes a desarrollar tanto la habilidad expresiva en el idioma extranjero como la habilidad de compresión mediante la escucha.

\section{Lo que hacemos}

Ahora bien, ¿qué es lo que tanto el estudiante como el profesor se sienten inclinados a hacer al iniciar una determinada lección y unidad y encarar la parte oral, pronunciación y comunicación, mediante un dialogo o minidiálogo?

\subsection{La parte de escucha (la lectura como escucha)}

a. En primer lugar se realiza la lectura del dialogo de la lección respectiva por parte del profesor (o se escucha desde el casete) haciendo énfasis en 
Revista Estudiantil HUELLAS. Año 5 No. 10. (Febrero, 1990). Lenguas Modernas, Universidad Mariana. San Juan de Pasto.

la pronunciación. Simultáneamente con la lectura del profesor, o escucha del casete, los estudiantes siguen mentalmente la LECTURA en el texto.

b. En segundo lugar, el profesor hace repetir a los estudiantes el diálogo en voz alta (ellos LEEN en realidad el diálogo del texto). A continuación, el profesor pregunta acerca del vocabulario nuevo y se explican los términos, expresiones o estructuras nuevas.

c. Después de esta etapa, el profesor realiza algunas preguntas de comprensión sobre el diálogo.

Seguidamente el profesor y los estudiantes se adentran a trabajar en la práctica de las estructuras con función comunicativa objeto de la lección. Es aquí donde tanto estudiantes como profesores se sienten tan inclinaos a hacer algo que parece lógico hacer, es decir, realizar los ejercicios o prácticas de conversación, como simples ejercicios de complementación de la lectura.

Describimos más detenidamente lo que se hace en cada caso:

\subsection{La parte del habla (el habla como lectura)}

a. El profesor escoge cada una de las actividades (las cuatro o cinco prácticas iniciales del texto) y prepara a sus estudiantes para trabajar en binas y desarrollar las prácticas. Los estudiantes realizan una especie de ejercicios de "llenar en los espacios en blanco".

b. En seguida, el profesor pide a sus estudiantes que desarrollen los ejercicios en forma oral para compararlos con los de los demás estudiantes. Estos leen del texto el desarrollo de los ejercicios.

En el momento de terminar la práctica y a lo largo de la misma parece que todo ha estado saliendo en forma normal. Sin embrago, los estudiantes no se han desprendido de sus textos un solo momento desde que se inició la práctica. Casi todo el trabajo de clase se ha basado en la lectura de los diálogos del texto.

\section{El problema}

Como decía al principio, nadie objetaría que el estudiante siga el texto y lo utilice con cualquier ejercicio, aún más, la mayoría de profesores alentaríamos al estudiante a que lo haga pensando que de esta forma el estudiantes está en realidad trabajando concentrado, desarrollando las practicas correctamente para él y los demás compañeros. Desafortunadamente esto no es así y la experiencia con este tipo de actividades nos puede contar (personalmente lo he constatado) 
Revista Estudiantil HUELLAS. Año 5 No. 10. (Febrero, 1990). Lenguas Modernas, Universidad Mariana. San Juan de Pasto.

que en realidad el estudiante está desperdiciando su tiempo y esfuerzo; además, frenando y limitando su capacidad para desarrollar una habilidad real de comunicación como expresión y compresión auditiva en el idioma extranjero.

¿En qué consiste en realidad el problema? Sencilla y llanamente en que todas las prácticas orales de comunicación y de escucha se han estado convirtiendo en una forma de compresión de lectura en voz alta (aunque suene contradictorio: 'comprensión de lectura de un diálogo').

De mi experiencia con buena cantidad de estudiantes de nivel medios, vocacionales y superiores he podido evidenciar que estudiantes de niveles intermedios y avanzados que han seguido como hábito el proceso descrito anteriormente, carecen de identidad de producción comunicativa oral en el idioma extranjero. Para ellos, el texto se ha convertido en una especie de "muleta" sin la cual no pueden "caminar" por si solos. Esto con graves deficiencias en la parte de expresión, espontaneidad, naturalidad y uso de las funciones comunicativas elementales (saludos, solicitud de informaciones, disculpas, sugerencias, etc.)

Los siguientes son ejemplos de expresiones, (con su respectiva reacción lingüística implícita), que no deberían ser leídas en una práctica oral comunicativa:

What do you do?

What would you like to do?

I'm from Pasto. What about you?

How are you today, Carlos?

$\mathrm{Hi}$, Jorge. How's everything!

Would you please, close that door.

I'm really sorry. Ma'am

Let's get something to eat

Say, who's that?

Etc... 
Revista Estudiantil HUELLAS. Año 5 No. 10. (Febrero, 1990). Lenguas Modernas, Universidad Mariana. San Juan de Pasto.

Ahora veamos algunos problemas a los que el estudiante se vería enfrentado por causa del uso inadecuado del texto guía y su extremada dependencia en la parte de producción oral.

\subsection{Pérdida de la concentración de escucha}

Es evidente que el estudiante que está escuchando al profesor o el casete maestro en la producción del diálogo y lo está leyendo al mismo tiempo del texto, no está haciendo uso de su capacidad auditiva de discriminación y captación auditivas sino que simplemente está siendo guiado (además en forma no muy confiable) de la forma escrita de la lengua, y esto en esta parte de la práctica de escucha no interesada y por el contrario produce más mal que bien. Nuestra capacidad de discriminación y captación de la escucha se pierde en la misma medida que se realiza la lectura del guion (entendemos no lo que escuchamos sino lo que está escrito, dos fuentes diferentes). Deberíamos en esta parte estar haciendo un esfuerzo por "leer" auditivamente los sonidos distintivos del idioma en cuestión para aprender a reconocerlos y procesarlos auditivamente.

Es necesario enfatizar el hecho de que a oír se aprende escuchando y no leyendo, ya que los estudiantes lo que están escuchando es su propia lectura "idealizada". Es como si quisiéramos aprender a hablar leyendo; por el contrario a hablar se aprende hablando y a escuchar, escuchando.

\subsection{Pérdida de captación de patrones fonéticos y suprasegmentales}

Al leer perdemos concentración sobre el énfasis que se desprenden de las diferentes expresiones en el diálogo: aspectos de pronunciación y entonación directos (sin que medie el texto escrito). El cambio se lo puede evidenciar cuando los estudiantes (sin tener que leer) están concentrados en hacer sentido de lo que escuchan, en la pronunciación, articulación y énfasis que hace el profesor al desarrollar el diálogo.

\subsection{Pérdida de fluidez}

Creo que no hay necesidad de demostrar que toda captación oral y procesamiento directo de la lengua es supremamente más rápida que de la que se puede hacer leyendo, y mucho más que la lectura en voz alta. Si al escuchar también leemos estamos siendo frenados por la forma escrita, es decir, por el texto. 
Revista Estudiantil HUELLAS. Año 5 No. 10. (Febrero, 1990). Lenguas Modernas, Universidad Mariana. San Juan de Pasto.

\subsection{Pérdida de naturalidad y espontaneidad}

Es indudable que una conversación por más elemental que sea debe incluir aspectos de naturalidad e inclusive espontaneidad al expresarse. Imagínese conversando con alguien que le contestara como si estuviera leyendo. Decididamente que si nos acostumbramos a leer la parte oral de la lengua, lo que integramos como habilidad oral (si hay alguna) sería por demás artificial; estaríamos dependiendo de un guion.

\subsection{Pérdida de creatividad}

Una de las características más importantes en la habilidad de la comunicación oral es la de ser creativos sobre todo cuando se trata de conversación formal (naturalidad y espontaneidad implícitas). Nadie hablaría informalmente teniendo un guion preparado para responder a preguntas como "How are you?".

Si nos basamos nuevamente en la lectura como "ayuda" para la parte oral, podríamos estar restringiendo nuestro repertorio lingüístico a una o dos formas de la amplia gama de respuestas creativas a determinada pregunta:

A. How are you, George?

B. - Fine, and you?

- I'm fine

- I'm OK

- Fine thanks

- Fine, and you?

- Not bad. What about you?

Inclusive:

- Ok

- Good

- Not too bad, etc.

\section{Lo que podríamos hacer}

A continuación me permito dar a conocer algunas pautas a seguir para enfrentar la parte de escucha y producción oral y con las que he podido evidenciar resultados halagadores. Tal vez no tanto debido a la idoneidad del profesor sino a que el estudiante obtiene un proceso visible para la realización y práctica de 
Revista Estudiantil HUELLAS. Año 5 No. 10. (Febrero, 1990). Lenguas Modernas, Universidad Mariana. San Juan de Pasto.

sus habilidades lingüísticas desarrollando al mismo tiempo estrategias de aprendizaje satisfactorias y adquiriendo seguridad.

\subsection{Escucha}

Como táctica a seguir y que recomiendo en la parte de la introducción a la historia o dialogo inicial de cada lección es que el estudiante haga todo menos abrir el texto si no hay otra fuente de entrada más que el diálogo; que el estudiante escuche ya sea al profesor o el casete maestro.

a. Los estudiantes escuchan atentamente al profesor realizar el diálogo en voz alta o escuchan el casete. Tratan de ubicar el contexto imaginándose lo que sucede en el diálogo.

b. Se repite nuevamente la parte de escucha para que se puedan aclarar dudas y que le idea del contexto del diálogo o historia quede identificado. El profesor debería enfatizar este aspecto para que los estudiantes hagan un esfuerzo cognitivo de discriminación de lo escuchado y progresivamente de su significado y su sentido. Esto hará que la escucha se realice con un propósito y no por el solo hecho de escuchar y de hacerlo en forma "pasiva".

\subsection{Repetición}

En la parte de repetición del diálogo por parte de los estudiantes, que generalmente se realiza después del profesor, los estudiantes todavía no deberían abrir los textos y repetir leyendo. Lo que en realidad sirve aquí es el acto de captar una cadena de sonidos, palabras, frases, etc. con fines de identificación en lo posible y procesarlos directamente (o al menos hacer el esfuerzo) para repetirlos sin que medie la lectura como ayuda visual.

La lectura del diálogo desde el texto en este paso estría engañando al profesor y a los estudiantes en el sentido de que se diría "qué bien que repiten los estudiantes" tomando lectura por captación y procesamiento lingüístico en memoria de corto plazo. En realidad se tendría que decir "qué bien que leen los estudiantes". Leyendo no estaríamos repitiendo lo que escuchamos sino simple y llanamente leyendo lo que el profesor ha hecho, la lectura. Realmente la repetición es este caso no necesariamente implicaría compresión, sin embargo el estudiante podría estar haciendo un esfuerzo auditivo y de retentiva para poder procesar una frase u oración que ha escuchado. Nuevamente, al repetir leyendo 
Revista Estudiantil HUELLAS. Año 5 No. 10. (Febrero, 1990). Lenguas Modernas, Universidad Mariana. San Juan de Pasto.

se perdería la capacidad de retención y de procesamiento directos que se realiza por medio de la escucha en una situación natural.

El profesor debe enfatizar el aspecto de la concentración de los estudiantes en lo que escuchan sea que haya o no haya compresión.

a. Realice la lectura para la repetición mediante unidades significativas (frases u oraciones). Al cortar una oración larga haga que se identifiquen las partes distinguibles de sentido. Ejemplo:

I'd like to have a large hamburger/ with tomato and lettuce.

b. Insista en que la repetición debe ser en voz alta y fuerte para procurar ejercitar los articuladores. Ojala se exagere en la pronunciación como mecanismo para adquirir los rasgos o patrones fonéticos, suprasegmentales y de entonación del inglés.

\subsection{Ejercicios orales}

En la práctica de los ejercicios para la producción oral con fines comunicativos donde se introducen y practican las estructuras en cuestión, hay que enfatizar el hecho de que se deben desarrollar oralmente sin demasiado apoyo del texto. El propósito de esto es para que los ejercicios no se conviertan en una práctica más de "llenar los espacios en blanco" para luego leerse en voz alta. Creo que aquí radica el éxito o fracaso de la parte comunicativa y habilidad oral y de escucha de la lengua.

Si las prácticas se realizan con base en una simple lectura de un ejercicio escrito de complementación, aunque se trate de minidiálogos de una o dos líneas el estudiante no podrá desprenderse del texto para realizarlos. El resultado será que cuando se le pregunte algo en forma natural, buscará en vano la respuesta en el texto. Lógicamente su orientación ha sido la lectura en vez del habla y la escucha. En este caso lo que se hace es crear en el estudiante una dependencia fuerte en texto y frustrar el desarrollo de su capacidad oral comunicativaauditiva, su espontaneidad y creatividad lingüísticas.

La experiencia que como profesor he tenido me ha brindado la oportunidad de atestiguar que no se concibe que un libro de texto para el aprendizaje del inglés como el de la serie "In Touch", de orientación funcional-comunicativa, destacándose la parte oral (a diferencia de textos tradicionales), no surta efecto en precisamente ese aspecto. Sin embargo, el problema no es directamente por 
Revista Estudiantil HUELLAS. Año 5 No. 10. (Febrero, 1990). Lenguas Modernas, Universidad Mariana. San Juan de Pasto.

causa del texto sino por su inadecuada aplicación y uso. No se consideraría normal que a la pregunta del profesor "what's your name" o "what do you do" o "what's your nationality", "what do you think about...", el estudiante busque el texto para encontrar la respuesta...

El estudiante debe comprender que los modelos de práctica en el texto sólo son una pauta o ejemplo en el que debería ayudarse para realizar la práctica utilizando su propia experiencia, contexto y conocimiento.

a. Diga a sus estudiantes que asuman el papel de interlocutores en la práctica (i. e. A: B: etc.) y que llenen los espacios en blanco pero ORALMENTE (sin necesidad de escribir en ellos) con la información de su propia experiencia. Este ejercicio debe realizarse al menos en grupos de dos y no se deben dejar individualmente para que el estudiante los complete en la casa en forma escrita y los olvide.

b. Los estudiantes realizan la práctica conjunta, ante los demás compañeros de clase en forma oral sin tener que leer del texto (a libro cerrado). Es importante enfatizar aquí que los estudiantes podrán aprender también al escuchar a los demás. Así que no es necesario que estén siempre pendientes del texto.

Teniendo en cuenta estas sugerencias, el estudiante tendría muchas más probabilidades de desarrollar tanto su capacidad oral como auditiva o de escucha en forma satisfactoria. De esta manera el carácter de aprendizaje del inglés en su forma oral como expresión comunicativa natural se incrementaría y los resultados no tardarían en evidenciarse. Desafortunadamente para empezar a hacer esto con estudiantes de un nivel intermedio o alto intermedio o en el peor de los casos, con los nivel avanzado que ya hayan estado habituados a leer las prácticas de comunicación y escucha del texto, el trabajo será mucho más difícil que empezar a hacerlo con principiantes, puesto que los primeros no aceptarían desprenderse tan fácilmente de la forma de dependencia del texto. De todas maneras, habría que hacerlo en forma remedial antes de empezar un curso.

\section{Conclusión}

Tal vez no haya mucho que concluir en este artículo aparte de aclarar que de ninguna manera estoy en contra de la lectura, por el contrario lo que he querido enfatizar aquí es el hecho de no tomar el desarrollo de una habilidad o aspecto 
Revista Estudiantil HUELLAS. Año 5 No. 10. (Febrero, 1990). Lenguas Modernas, Universidad Mariana. San Juan de Pasto.

lingüístico por otro, es decir hacer comprensión de lectura de los diálogos a expensas de la comprensión de escucha y la producción oral.

Espero, con este artículo, haber contribuido en algo a la inquietud de algunos colegas de bachillerato que me han preguntado acerca de este aspecto y de posibles soluciones y recomendaciones. 